\title{
Evaluation of the real-world impact of rotational thromboelastometry-guided transfusion protocol in patients undergoing proximal aortic surgery
}

\author{
Samuel St-Onge, MD, ${ }^{a}$ Émile Lemoine, MD, ${ }^{a}$ Ismail Bouhout, MD, MSc, ${ }^{a}$ Antoine Rochon, MD, \\ Ismail El-Hamamsy, $\mathrm{MD}, \mathrm{PhD},{ }^{\mathrm{a}}$ Yoan Lamarche, $\mathrm{MD}, \mathrm{MSc},{ }^{\mathrm{a}}$ and Philippe Demers, $\mathrm{MD}, \mathrm{MSc}^{\mathrm{a}}$
}

\section{ABSTRACT}

Background: Complex aortic procedures are potentially associated with important blood loss and coagulopathy. The aim of this study was to assess the impact of rotational thromboelastometry (ROTEM, Tem International GmBH, Munich, Germany) on transfusion requirements after proximal aortic operations in a real-world setting.

Methods: This single-center retrospective analysis based on 385 consecutive patients undergoing cardiac surgeries involving the aortic root, ascending aorta, or aortic arch compared 197 controls managed according to routine transfusion protocol before the introduction of the ROTEM in 2012 with 188 patients operated afterward. With the use of a 1:1 propensity score match, 224 patients were included in paired analysis (112 in each group). The primary end point was erythrocytes transfusion rate. The secondary end points comprised the transfusion of other allogeneic blood products, number of units transfused, postoperative blood loss, massive transfusion rate, and use of other hemostatic products.

Results: ROTEM implementation was associated with a trend toward reduction in the rate of erythrocytes transfusion $(57 \%$ vs $46 \%, P=.08)$ and a decreased median number of units transfused for erythrocytes (1.0 [0.0-4.0] unit vs 0.0 [0.0-2.0] unit, $P=.03$ ) and plasma (0.0 [0.0-4.0] unit vs $0.0[0.0-2.0]$ unit, $P=.04)$. After sensitivity analysis, ROTEM displayed a comparable rate of erythrocytes transfusion ( $58 \%$ vs $47 \%, P=.15)$.

Conclusions: In a real-world setting, ROTEM-based algorithm implementation could help reduce excess erythrocytes transfusion for complex aortic procedures. We advocate for a strict adherence and concerted team effort to maximize the benefits of such addition to patients' management. (J Thorac Cardiovasc Surg 2019;157:1045-54)

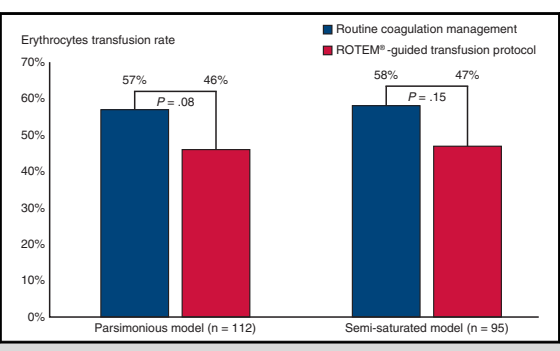

In a real-world setting, ROTEM could reduce erythrocytes transfusion.

Central Message

Provided a strict implementation, the ROTEMguided protocol may prevent excess erythrocytes transfusions after proximal aortic surgery.

\section{Perspective}

Important perioperative bleeding often complicates complex aortic procedures. We evaluated a ROTEM-based transfusion protocol in a real-world setting and demonstrated the potential to reduce erythrocytes transfusion, mainly in terms of the absolute number of RBC units needed postoperatively. Our findings highlight the importance of a strict adherence to the protocol to effectively minimize patients' exposure to allogeneic blood products.

See Commentaries on pages 1055 and 1058 .
Complex aortic procedures are often complicated by impaired hemostasis and important bleeding. ${ }^{1}$ Hemodilution and consumption of coagulation factors induced by cardiopulmonary bypass (CPB), deep hypothermia, hyperfibrinolysis, ischemia-reperfusion injury, activation

\footnotetext{
From the Departments of ${ }^{\mathrm{a}}$ Cardiac Surgery and ${ }^{\mathrm{b}}$ Anesthesia, Montreal Heart Institute, Université de Montréal School of Medicine, Montreal, Quebec, Canada.

Received for publication Dec 19, 2016; revisions received June 12, 2018; accepted for publication July 2, 2018; available ahead of print Sept 6, 2018.

Address for reprints: Philippe Demers, MD, MSc, 5000 Belanger St, Montreal, Quebec, Canada H1T1C8 (E-mail: p.demers@umontreal.ca). $0022-5223 / \$ 36.00$

Copyright (c) 2018 by The American Association for Thoracic Surgery https://doi.org/10.1016/j.jtcvs.2018.07.043
}

of systemic inflammatory response, extensive tissue trauma, and prior antiplatelet or anticoagulant therapy have all been reported as contributing factors to the hemostatic alterations observed after aortic surgery. ${ }^{2,3}$ Although significant perioperative blood loss is independently associated with increased morbidity and in-hospital mortality, major postoperative complications can also directly result

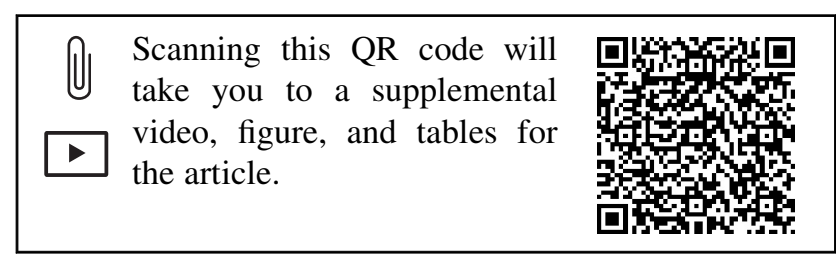




$$
\begin{aligned}
& \text { Abbreviations and Acronyms } \\
& \begin{aligned}
\text { ACT } & =\text { activated clotting time } \\
\text { CPB } & =\text { cardiopulmonary bypass } \\
\text { DDAVP } & =\text { desmopressin } \\
\text { FFP } & =\text { fresh-frozen plasma } \\
\text { ICU } & =\text { intensive care unit } \\
\text { OR } & =\text { operating room } \\
\text { PCC } & =\text { prothrombin complex concentrate } \\
\text { POC } & =\text { point-of-care } \\
\text { rFVIIa } & =\text { recombinant factor VIIa } \\
\text { ROTEM } & =\text { rotational thromboelastography } \\
\text { RBC } & =\text { red blood cell }
\end{aligned}
\end{aligned}
$$

from allogeneic blood product transfusions, such as transfusion-related immunomodulation, acute lung injury, and volume overload. ${ }^{4-9}$ Therefore, strategies aimed at minimizing blood loss and the need for transfusions are an important component of complex cardiac procedures that include an inherently higher risk of postoperative bleeding. $3,4,6,10,11$

Point-of-care (POC) testing with rotational thromboelastometry (ROTEM; Tem International $\mathrm{GmBH}$, Munich, Germany) allows clinicians to better determine which components of hemostasis are abnormal and therefore most likely to be responsible for bleeding. This not only is achieved in a shorter time lapse than standard coagulation testing but also allows for more specific and goaldirected interventions. ${ }^{12-15}$ However, these benefits so far have been demonstrated only in the strictly enforced setting of randomized control trials. ${ }^{16,17}$ After a thorough review of literature, we found no study that has tried to document these benefits on complex aortic procedures in a real-world setting, where physicians are allowed to deviate from the protocol at any point in their intervention. Assessing the real-world impact of this tool could help centers evaluate its true benefits and weigh it against the potential downfalls of its implementation into their surgical teams.

The objective of the present study is to evaluate the impact of the implementation of a ROTEM-based transfusion algorithm for bleeding management after surgical procedures involving the proximal aorta in a real-world setting and assess how and to what extent this new protocol can constitute an improvement over routine practice.

\section{MATERIAL AND METHODS \\ Study Population}

This is a retrospective analysis of prospectively collected patient data that was undertaken at the Montreal Heart Institute. After approval from the review board, our institutional surgical database was queried to identify all consecutive patients who underwent aortic procedures involving the root, ascending aorta, or aortic arch in the period before and after the implementation of a ROTEM-based transfusion algorithm in
2012. Interventions included elective or emergency ascending aortic replacement, aortic root procedures (remodeling, reimplantation, Ross and Bentall procedures), and partial or complete arch replacement. There was no exclusion criterion. The study population comprised a total of 385 consecutive patients undergoing aortic surgery from 2011 to 2014 . A total of 197 patients managed according to routine perioperative bleeding management strategy from March 2011 to October 2012 were compared with 188 patients managed according to the newly implemented ROTEM-based algorithm between October 2012 and June 2014. Patients were matched into 115 pairs according to a propensity score matching based on baseline and operative characteristics to alleviate the effects of measurable cofounders. The time frame for the 2 groups was set to be of similar length ( $~ 600$ days) before and after the introduction of ROTEM in our institution.

\section{Outcomes}

All blood products reported were given after protamine administration, the time at which the first ROTEM analysis would occur. The primary outcome was red blood cell (RBC) transfusion rate. Secondary outcomes comprised other allogeneic blood components (fresh-frozen plasma [FFP], platelets, and cryoprecipitate); transfusion rates; the number of units of RBC, perfluorocarbon, and platelets transfused; postoperative blood loss at 12 and 24 hours; exposure to 1 or more allogeneic blood product; total volume of transfused allogeneic blood products; massive transfusion rates; and the use of alternative hemostatic products (prothrombin complex concentrate [PCC], desmopressin [DDAVP], and recombinant factor VIIa [rFVIIa]). Massive transfusion was defined as the use of more than 20 cumulative units of RBC, perfluorocarbon, and platelets. ${ }^{16}$ Of note, cryoprecipitate and platelets transfused were reported as multiple exposures according to the number of donors per pool. In addition, clinical outcomes were also analyzed with an emphasis on reexploration for bleeding, ventilation time, intensive care unit (ICU) length of stay, neurologic events, and renal complications.

\section{Intraoperative Management}

The surgical team throughout the study period was formed by 10 senior surgeons, with 3 of them having a practice mostly dedicated to aortic interventions. When an elective surgery was performed, antiplatelet therapy was discontinued for 7 days before surgery when feasible. All patients receiving anticoagulation therapy were bridged by heparin after discontinuation of oral anticoagulant for 5 or more days. There were no major changes in the $\mathrm{CPB}$ practices throughout the study period. Systemic full-dose heparin $(300 \mathrm{U} / \mathrm{kg}$ ) was administered as a bolus before cannulation for $\mathrm{CPB}$. The activated clotting time (ACT) was checked every 20 minutes during $\mathrm{CPB}$, and additional heparin (5000 units) was given until the ACT was greater than 480 seconds. Tranexamic acid was routinely used in both study arms according to a standardized protocol: $1 \mathrm{~g}$ before CPB, $500 \mathrm{mg}$ when initiating $\mathrm{CPB}$, followed by $400 \mathrm{mg}$ per hour until termination of $\mathrm{CPB} .{ }^{18} \mathrm{CPB}$ modalities varied according to surgeons' preferences and the clinical situation. Arterial cannulation site was selected by the attending surgeon on the basis of the type and extent of surgery. Hypothermic circulatory arrest with or without selective antegrade cerebral perfusion was used in cases involving the aortic arch and was deep $\left(14.1^{\circ} \mathrm{C}-20^{\circ} \mathrm{C}\right)$ or moderate $\left(20.1^{\circ} \mathrm{C}-28^{\circ} \mathrm{C}\right)$ based on nasopharyngeal temperature. Other procedures were mainly performed under mild $\left(28.1^{\circ} \mathrm{C}-34^{\circ} \mathrm{C}\right)$ hypothermia. Selective antegrade cerebral perfusion at $10 \mathrm{~mL} / \mathrm{kg} / \mathrm{min}$ was used through perfusion of the axillary artery. Distal aortic repair was performed with an open technique during hypothermic cardiac arrest. Perfusion was restored, and rewarming was initiated thereafter. Rewarming was discontinued once the nasopharyngeal temperature reached $36^{\circ} \mathrm{C}$. After discontinuation of $\mathrm{CPB}$, heparin was neutralized with protamine (ratio of $1: 1 ; 1 \mathrm{mg}$ of protamine per 100 units total heparin). An intraoperative cell-salvage device with reinfusion of 
shed mediastinal blood was used in all cases. Topical hemostatic agents were rarely used.

Triggers for $\mathrm{RBC}$ transfusion during $\mathrm{CPB}$ were identical in both groups: hematocrit less than $20 \%$ during $\mathrm{CPB}$ and hemoglobin less than 7.5 to $8.0 \mathrm{~g} / \mathrm{dL}$ after CPB. RBCs were occasionally transfused despite hemoglobin levels greater than $8.0 \mathrm{~g} / \mathrm{dL}$ when physiologic transfusion triggers (eg, $\mathrm{ScvO}_{2}<70 \%$ ) occurred. Hemoglobin measures were obtained via POC blood gas analyzers available in every operating room (OR).

\section{ROTEM-Based Algorithm}

The principles of ROTEM have been described. ${ }^{12,13,16}$ Our ROTEM-based algorithm includes a complete blood count, an arterial blood gas, and the following 4 different ROTEM tests: EXTEM and INTEM for evaluation of extrinsic and intrinsic coagulation pathways, HEPTEM for detection of un-neutralized heparin, and FIBTEM for identification of fibrinogen deficiency or fibrin polymerization disorder. Clotting time (seconds) and clot formation time (seconds) were recorded

\section{1}

Significant blood loss
$(\geq 300 \mathrm{ml} / \mathrm{h}$ and/or medico-surgical consensus $)$
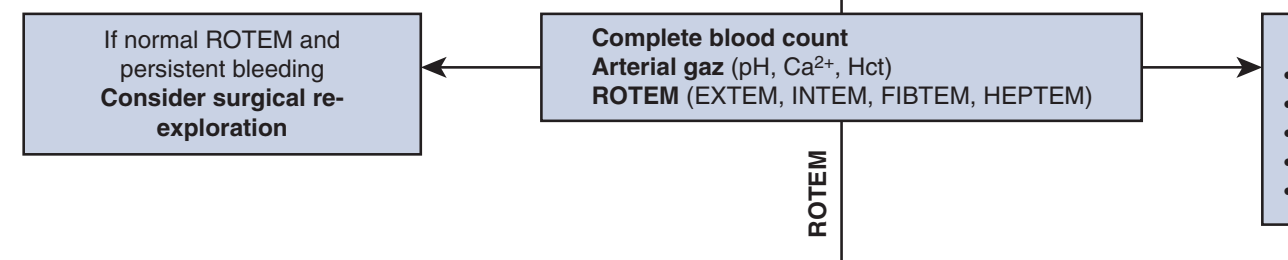

Correct and treat - Hypothermia $\left(\mathrm{T} \geq 35^{\circ} \mathrm{C}\right)$ - Hypocalcemia (> $1.03 \mathrm{mmol} / \mathrm{l})$

- Acidosis $(\mathrm{pH}>7.35)$

- Anemia (Hct $\geq 22 \%$ )

- Hypertension (MAP 55-65 mmHg)
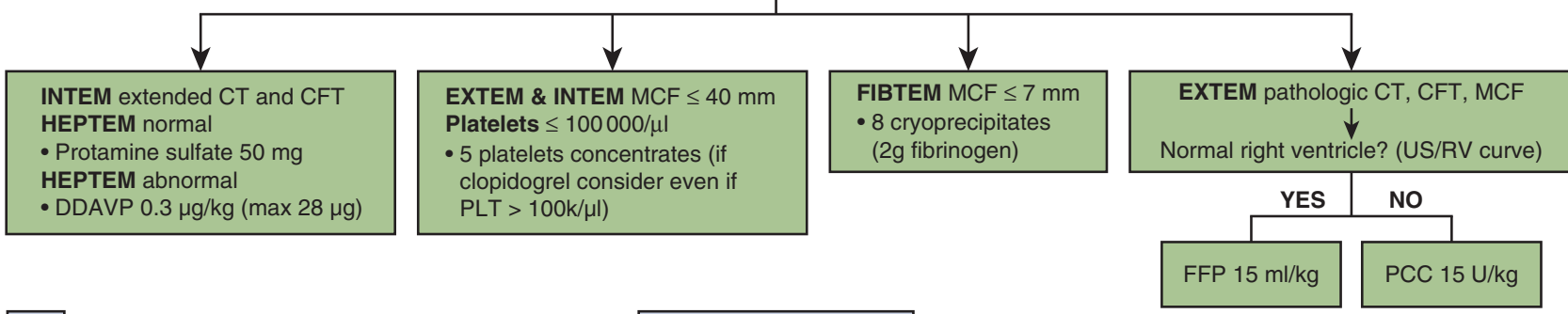

2

Unceasing hemorrhage
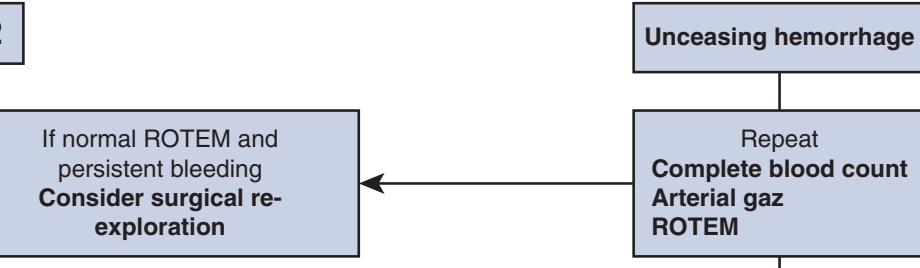

Arterial gaz

exploration

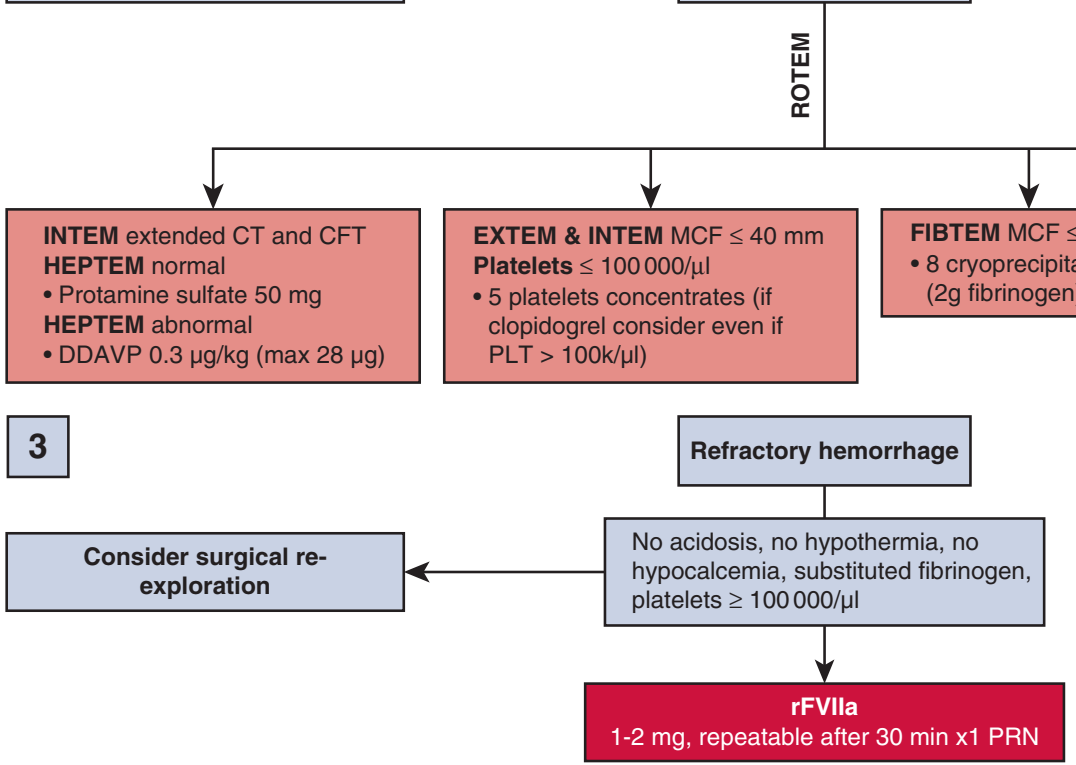

FIGURE 1. ROTEM-based (Tem International GmBH, Munich, Germany) transfusion algorithm. ROTEM, Rotational thromboelastography; $M A P$, mean arterial pressure; $C T$, clotting time; $C F T$, clot formation time; $D D A V P$, desmopressin; $M C F$, maximum clot firmness; $P L T$, platelets; $F F P$, fresh-frozen plasma; $P C C$, prothrombin complex concentrate; $r F V I I a$, recombinant factor VIIa; $R V$, right ventricle; $U S$, ultrasound. 
for INTEM, EXTEM, and HEPTEM, whereas maximum clot firmness $(\mathrm{mm})$ was recorded for INTEM, EXTEM, and FIBTEM.

The algorithm guiding the bleeding management following the ROTEM testing is illustrated in Figure 1. Initial ROTEM analysis was performed after discontinuation of $\mathrm{CPB}$ and administration of protamine. Test results were available to the OR personnel 10 minutes after blood sampling. The required blood products were ordered according to the transfusion protocol and transfused immediately. The extent of bleeding was assessed subjectively by the attending surgeon as the absence of visible clots in the surgical field 15 minutes after administration of blood products. A second round of tests (complete blood count, arterial blood gas, and ROTEM analysis) was carried out if hemostasis was still unsatisfactory. When hemostasis was deemed adequate, median sternotomy was closed and the patient was transferred to the ICU. During the first 24 hours, further ROTEM tests were performed in the ICU only in case of recurrent bleeding ( $>200 \mathrm{~mL}$ in the first hour or $>100 \mathrm{~mL} / \mathrm{h}$ thereafter). Further blood products were given according to the results of ROTEM analysis. In cases of persistent bleeding in the OR, ROTEM coagulation analysis was performed 15 minutes after administration of all appropriate coagulation products, and new blood products were ordered accordingly. When all preceding strategies failed, rFVIIa was administered. Principles behind the ROTEM analysis and our ROTEM-based algorithm are shown in Video 1.

\section{Routine Coagulation Management}

Patients in the control group received transfusions in the OR on the basis of clinical judgment and standard coagulation test results. After discontinuation of $\mathrm{CPB}$, protamine was administered first, and other blood products were transfused afterward, if the attending anesthesiologist and operating surgeon deemed them necessary. One additional dose of protamine $(50 \mathrm{mg})$ was given if the postprotamine ACT had not returned to within $15 \%$ of the preheparin value. Blood samples for laboratory tests were drawn thereafter. If no abnormal bleeding was observed, the chest was closed, and further blood products were transfused only in cases of increased bleeding in the ICU $(>200 \mathrm{~mL}$ in the first hour or $>100 \mathrm{~mL} / \mathrm{h}$ thereafter). In cases of persistent bleeding with no visible clots in the operating field, further blood products were administered on an empirical basis until coagulation test results were available. When all preceding strategies failed, rFVIIa was administered to control the bleeding. A time interval of approximately 50 minutes was required to receive coagulogram results from our laboratory.

Trigger for platelets transfusion was a platelet count less than 100 cells $/ \mu \mathrm{L}$, although transfusion occurred irrespective to this threshold when platelet dysfunction was suspected (ie, prolonged CPB or recent antiplatelet drug use). Plasma was transfused if international normalized ratio was greater than 1.5 and cryoprecipitates (8-10 units) if fibrinogen level was less than $100 \mathrm{mg} / \mathrm{dL}$. Prothrombin complex concentrate (Beriplex; CSL Behring, King of Prussia, Pa) was administered instead of perfluorocarbon in cases of volume overload or right ventricular failure. ${ }^{19}$

\section{Statistical Analysis}

A logistic regression predicting the choice of transfusion algorithm (routine vs ROTEM-based) was conducted for all baseline and operative characteristics presented in Tables 1 and 2. A stepwise variable selection with an inclusion criterion of $P$ value .2 or less and retention criterion of $P$ value .1 or less was in the final model $(C$-statistic $=0.7)$. Seven variables significantly different between usual and ROTEM-based coagulation management in the cohort were identified. A parsimonious propensity score was computed to match the ROTEM group with the routine group according to coronary artery disease, fibrinogen, hemoglobin, and lactate levels before surgery, minimal perioperative temperature, Ross procedures, and hemoglobin nadir per procedure. Patients were matched $(1: 1)$ using the nearest neighbor method without

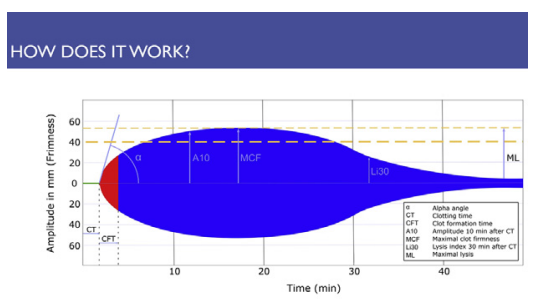

VIDEO 1. ROTEM, an interesting addition to our management of postoperative coagulopathy after complex aortic procedures. Video available at: https://www.jtcvs.org/article/S0022-5223(18)32022-1/fulltext.

replacement and a caliper width of 0.2 of the standard deviation of the logit of the estimated propensity score. The mean distance in the estimated propensity scores was $3.5 \% \pm 5.8 \%$. Covariance balance plots before and after propensity matching have been included (Figure E1). In a sensitivity analysis, a semisaturated propensity score matching including all variables in Tables 1 and 2 was also presented (Tables E1 and E2).

Continuous variables were compared using independent $t$ tests and presented as mean \pm standard deviation unless normality was ruled out using the Kolmogorov-Smirnov test. In such cases, the Mann-Whitney $U$ test was used and data were expressed as median and interquartile ranges. Discrete variables were presented as frequency (\%) and compared using the chi-square or Fisher exact test as appropriate. Within the matched pairs, comparison of continuous variables used paired $t$ tests for symmetrically distributed variables and Mann-Whitney $U$ test for skewed ones as categoric variables were compared using the McNemar test. Patient and operative characteristics were also compared using standardized differences. A difference greater than $20 \%$ was considered significant. Variables measured at different points in time were corrected for repeated measures. Missing data accounted for less than $1 \%$ of all collected information: They were imputed using the mean value of the entire cohort. All analyses were performed with SPSS v. 23.0 (IBM Corp, Armonk, NY).

\section{RESULTS}

\section{Baseline and Operative Characteristics}

In the unmatched cohort, more patients underwent deep hypothermic arrest in the routine group $(5 \%$ vs $1 \%$, $P=.01)$. The ROTEM group presented a higher proportion of Ross procedures ( $6 \%$ vs $11 \%, P=.07)$ and longer aortic crossclamp times (82.0 [57.5-116] minutes vs 89.0 [63.0-134] minutes, $P=.06$ ), but neither of these differences reached statistical significance. Furthermore, hemostasis time and allogeneic blood transfusion rates before protamine administration were similar in both groups.

After propensity score matching, the cohort comprised a total of 224 patients who were transfused according to routine coagulation management or ROTEM-guided transfusion protocol. Demographic (Table 1) and operative characteristics (Table 2) remained relatively unbalanced: Although none of the variables had a standardized difference greater than $20 \%, 14$ remained greater than $10 \%$. 
TABLE 1. Baseline characteristics

\begin{tabular}{|c|c|c|c|c|c|c|c|c|}
\hline \multirow[b]{2}{*}{ Variable } & \multicolumn{4}{|c|}{ Unadjusted data } & \multicolumn{4}{|c|}{ Propensity score-matched data } \\
\hline & $\begin{array}{c}\text { Routine } \\
(\mathrm{N}=197)\end{array}$ & $\begin{array}{c}\text { ROTEM } \\
\text { (Tem International } \\
\text { GmBH, Munich, } \\
\text { Germany) } \\
(\mathbf{N}=\mathbf{1 8 8})\end{array}$ & $\begin{array}{l}\text { Std } \\
\text { Diff }\end{array}$ & $\begin{array}{c}P \\
\text { value }\end{array}$ & $\begin{array}{c}\text { Routine } \\
(\mathrm{N}=\mathbf{1 1 2})\end{array}$ & $\begin{array}{c}\text { ROTEM } \\
(\mathrm{N}=112)\end{array}$ & $\begin{array}{l}\text { Std } \\
\text { Diff }\end{array}$ & $\begin{array}{c}P \\
\text { value }\end{array}$ \\
\hline Age (y) & $57.3 \pm 16.1$ & $58.6 \pm 14.4$ & 8.0 & .42 & $57.6 \pm 15.8$ & $58.7 \pm 14.3$ & 8.0 & .59 \\
\hline Male & $138(70)$ & $135(72)$ & 4.0 & .70 & $84(75)$ & $76(68)$ & -9.0 & .24 \\
\hline BMI $\left(\mathrm{kg} / \mathrm{m}^{2}\right)$ & $28.5 \pm 6.4$ & $27.9 \pm 5.5$ & -10.0 & .33 & $28.5 \pm 6.4$ & $28.2 \pm 5.9$ & -4.2 & .36 \\
\hline Arterial hypertension & $116(59)$ & $100(53)$ & -11.5 & .26 & $63(56)$ & $59(53)$ & -6.6 & .59 \\
\hline Diabetes mellitus & $22(11)$ & $28(15)$ & 11.1 & .28 & $14(13)$ & $12(11)$ & -2.4 & .68 \\
\hline Coronary artery disease & $47(24)$ & $52(28)$ & 8.7 & .39 & $29(26)$ & $27(24)$ & 0.0 & .76 \\
\hline Peripheral arterial disease & $12(6)$ & $15(8)$ & 7.4 & .47 & $7(6)$ & $10(9)$ & 6.4 & .45 \\
\hline Hepatopathy & $5(3)$ & $7(4)$ & 6.8 & .50 & $3(3)$ & $3(3)$ & 14.0 & $>.99$ \\
\hline COPD & $24(12)$ & $23(12)$ & 0.1 & .99 & $11(10)$ & $14(13)$ & 5.0 & .52 \\
\hline Dyslipidemia & $91(46)$ & $81(43)$ & -6.3 & .54 & $47(42)$ & $42(38)$ & -4.9 & .50 \\
\hline Ascending aorta aneurysm & $163(83)$ & $155(82)$ & -0.8 & .94 & $94(84)$ & $95(85)$ & 4.4 & .85 \\
\hline Acute type A aortic dissection & $18(9)$ & $26(14)$ & 14.8 & .15 & $9(8)$ & $13(12)$ & 12.9 & .37 \\
\hline Chronic aortic dissection & $5(3)$ & $8(4)$ & 9.5 & .41 & $3(3)$ & $5(5)$ & 9.0 & .72 \\
\hline Endocarditis & $3(2)$ & $4(2)$ & 4.5 & .72 & $3(3)$ & $0(0)$ & 18.3 & .25 \\
\hline Preoperative creatinine $(\mathrm{mg} / \mathrm{dL})$ & $0.87(0.77-1.04)$ & $0.91(0.74-1.03)$ & 14.4 & .66 & $0.88(0.78-1.04)$ & $0.91(0.72-1.03)$ & 5.3 & .69 \\
\hline Chronic kidney disease & $6(3)$ & $10(5)$ & 11.4 & .26 & $4(4)$ & $6(5)$ & 8.1 & $>.99$ \\
\hline \multicolumn{9}{|l|}{ Medication } \\
\hline Aspirin & $67(34)$ & $55(29)$ & -10.2 & .32 & $28(30)$ & $34(36)$ & 14.1 & .34 \\
\hline Heparin & $37(19)$ & $28(15)$ & -10.4 & .31 & $17(15)$ & $12(11)$ & -17.4 & .32 \\
\hline Warfarin & $31(16)$ & $22(12)$ & -11.7 & .25 & $12(13)$ & $16(17)$ & 11.9 & .40 \\
\hline Discontinued antiplatelet therapy & $31(16)$ & $31(16)$ & 0.8 & .93 & $9(8)$ & $10(9)$ & 0.5 & .84 \\
\hline \multicolumn{9}{|l|}{ Coagulation testing } \\
\hline INR $>1.2 \mathrm{~s}$ & $16(8)$ & $18(10)$ & 5.1 & .62 & $7(7)$ & 7 (7) & -1.7 & $>.99$ \\
\hline PTT $>35 \mathrm{~s}$ & $16(8)$ & $16(9)$ & 1.4 & .89 & $10(9)$ & $8(7)$ & -5.9 & .62 \\
\hline Fibrinogen (mg/dL) & $365 \pm 82.2$ & $418 \pm 121$ & 6.2 & $<.01$ & $385 \pm 93.6$ & $373 \pm 75.2$ & -2.9 & .29 \\
\hline \multicolumn{9}{|l|}{ Hemogram } \\
\hline Hematocrit (\%) & $39.9 \pm 4.3$ & $39.4 \pm 4.6$ & -11.2 & .27 & $39.7 \pm 4.6$ & $40.0 \pm 4.0$ & 9.6 & .42 \\
\hline $\mathrm{Hb}(\mathrm{g} / \mathrm{dL})$ & $13.7 \pm 1.6$ & $13.3 \pm 1.6$ & -21.7 & .03 & $13.6 \pm 1.7$ & $13.8 \pm 1.4$ & 12.3 & .17 \\
\hline Platelets (cells $/ \mu \mathrm{L})$ & $194.5 \pm 55.1$ & $194.8 \pm 62.8$ & 0.5 & .96 & $189.3 \pm 53.4$ & $185.3 \pm 55.8$ & -9.8 & .57 \\
\hline Lactates (mmol/L) & $0.8(0.6-1.1)$ & $0.9(0.7-1.2)$ & 23.7 & $<.01$ & $0.9(0.6-1.2)$ & $0.9(0.7-1.1)$ & 3.1 & .51 \\
\hline euroSCORE II (\%) & $7.38 \pm 7.71$ & $7.04 \pm 7.54$ & -4.4 & .83 & $6.93 \pm 6.67$ & $6.56 \pm 6.36$ & -6.1 & .34 \\
\hline
\end{tabular}

Categoric variables are presented as frequency (\%); continuous variables are presented as mean \pm SD when they were normally distributed or as median (IQR) otherwise. ROTEM, Rotational thromboelastography; Std Diff, standardized difference; $B M I$, body mass index; COPD, chronic obstructive pulmonary disease; INR, international normalized ratio; PTT, partial thromboplastin time; $H b$, hemoglobin; euroSCORE, euroSCORE, European System for Cardiac Operative Risk Evaluation.

\section{Primary End Point}

Details of perioperative blood loss and exposure to allogeneic blood and alternative hemostatic products are displayed in Table 3. Within the unmatched cohort, patients managed under the ROTEM-guided protocol had statistically significant lower rates of RBC transfusions $(59 \%$ vs $48 \%, P=.03)$ when compared with patients transfused according to routine coagulation protocol.
In the matched cohort, there was a lower use of RBCs in the ROTEM group (58\% vs $47 \%$ ). However, this result did not reach statistical significance $(P=.08)$.

\section{Secondary End Points}

After propensity matching, ROTEM was significantly associated with less units of RBCs (1.0 [0.0-4.0] unit vs $0.0[0.0-2.0]$ unit, $P=.03)$ and FFP $(0.0$ [0.0-4.0] 
TABLE 2. Operative characteristics

\begin{tabular}{|c|c|c|c|c|c|c|c|c|}
\hline \multirow[b]{2}{*}{ Variable } & \multicolumn{4}{|c|}{ Unadjusted data } & \multicolumn{4}{|c|}{ Propensity score-matched data } \\
\hline & $\begin{array}{c}\text { Routine } \\
(\mathbf{N}=197)\end{array}$ & $\begin{array}{c}\text { ROTEM } \\
(\mathbf{N}=\mathbf{1 8 8})\end{array}$ & $\begin{array}{l}\text { Std } \\
\text { Diff }\end{array}$ & $\begin{array}{c}P \\
\text { value }\end{array}$ & $\begin{array}{c}\text { Routine } \\
(\mathrm{N}=112)\end{array}$ & $\begin{array}{c}\text { ROTEM } \\
(\mathbf{N}=112)\end{array}$ & SD & $\begin{array}{c}P \\
\text { value }\end{array}$ \\
\hline Elective procedure & $156(79)$ & $149(79)$ & 0.2 & .99 & $86(77)$ & $94(84)$ & 14.1 & .18 \\
\hline Previous cardiac surgery & $42(21)$ & $33(18)$ & -9.5 & .35 & $19(17)$ & $22(20)$ & 4.1 & .60 \\
\hline CPB time (min) & $122(91.0-170)$ & $128(95.0-184)$ & 8.9 & .26 & $125(101-171)$ & $122(91.0-165)$ & -8.8 & .47 \\
\hline Aortic crossclamp time (min) & $82.0(57.5-116)$ & $89.0(63.0-134)$ & 16.9 & .06 & $86.9(63.0-119)$ & $86.5(58.0-128)$ & -3.7 & .87 \\
\hline Hemostasis time (min) & $55.0(44.0-83.5)$ & $58.0(47.0-80.0)$ & 5.2 & .22 & $55.5(45.0-76.5)$ & $56.5(46.0-76.8)$ & 5.9 & .71 \\
\hline Right axillary perfusion & $19(10)$ & $26(14)$ & 13.0 & .20 & $8(7)$ & $14(13)$ & 17.9 & .18 \\
\hline Femoral perfusion & $14(7)$ & $17(9)$ & -3.0 & .49 & $8(7)$ & $7(6)$ & -7.1 & .79 \\
\hline Circulatory arrest & $68(35)$ & $61(32)$ & -4.4 & .67 & $37(33)$ & $30(27)$ & -10.4 & .79 \\
\hline Circulatory arrest time (min) & $15.3 \pm 10.6$ & $13.6 \pm 7.3$ & 10.3 & .32 & $15.6 \pm 12.2$ & $14.1 \pm 7.6$ & -15.1 & .56 \\
\hline \multicolumn{9}{|l|}{ Hypothermia } \\
\hline $\operatorname{Mild}\left(28.1^{\circ} \mathrm{C}-34^{\circ} \mathrm{C}\right)$ & $135(69)$ & $122(65)$ & -5.9 & .45 & $79(71)$ & $77(69)$ & -3.5 & .77 \\
\hline Moderate $\left(20.1^{\circ} \mathrm{C}-28^{\circ} \mathrm{C}\right)$ & $50(25)$ & $58(31)$ & 14.6 & .23 & $28(25)$ & $30(27)$ & 7.6 & .76 \\
\hline Deep $\left(14.1^{\circ} \mathrm{C}-20^{\circ} \mathrm{C}\right)$ & $12(6)$ & $8(4)$ & -4.7 & .42 & $5(5)$ & $5(5)$ & 0.0 & $>.99$ \\
\hline Antegrade cerebral perfusion & $18(9)$ & $18(10)$ & 15.0 & .88 & $9(8)$ & $9(8)$ & 2.8 & $>.99$ \\
\hline AAR & $126(64)$ & $121(64)$ & -0.6 & .99 & $70(63)$ & $71(63)$ & 1.1 & .89 \\
\hline AAR alone & $8(4)$ & $4(2)$ & -11.2 & .38 & $3(3)$ & $4(4)$ & 4.7 & $>.99$ \\
\hline $\mathrm{AAR}+\mathrm{AVR}$ & $66(34)$ & $58(31)$ & -5.7 & .58 & $37(33)$ & $35(31)$ & -1.8 & .78 \\
\hline Aortic root procedure & $69(35)$ & $66(35)$ & -2.5 & .99 & $40(36)$ & $41(37)$ & 2.7 & .89 \\
\hline Bentall & $50(25)$ & $41(22)$ & -8.4 & .41 & $29(26)$ & $22(20)$ & -17.3 & .27 \\
\hline Yacoub & $11(6)$ & $15(8)$ & 9.5 & .35 & $6(5)$ & $11(10)$ & 16.9 & .21 \\
\hline Tirone David & $8(4)$ & $10(5)$ & 5.9 & .56 & $5(5)$ & $8(7)$ & 7.7 & .39 \\
\hline Ross & $11(6)$ & $20(11)$ & 13.8 & .07 & $9(8)$ & $9(8)$ & 2.4 & $>.99$ \\
\hline Total arch replacement & $4(2)$ & $7(4)$ & 10.1 & .37 & $4(4)$ & $3(3)$ & 0.0 & $>.99$ \\
\hline Hemiarch replacement & $64(32)$ & $54(29)$ & -8.2 & .42 & $33(30)$ & $27(24)$ & -10.7 & .37 \\
\hline Concomitant CABG & $36(18)$ & $44(23)$ & 12.7 & .22 & $24(21)$ & $19(17)$ & -4.0 & .40 \\
\hline \multicolumn{9}{|l|}{ Transfusion before protamine } \\
\hline $\mathrm{RBC}$ & $28(15)$ & $30(15)$ & 1.1 & .93 & $13(12)$ & $16(14)$ & 4.3 & .55 \\
\hline FFP & $2(1)$ & $3(2)$ & 2.7 & .62 & $1(1)$ & $1(1)$ & 0.0 & $>.99$ \\
\hline Platelets & $1(<1)$ & $2(1)$ & 1.3 & .54 & $0(0)$ & $2(2)$ & 3.8 & .50 \\
\hline Cryoprecipitate & $0(0)$ & $0(0)$ & 0.0 & $>.99$ & $0(0)$ & $0(0)$ & 0.0 & $>.99$ \\
\hline $\mathrm{Hb}$ nadir per procedure $(\mathrm{g} / \mathrm{dL})$ & $8.3 \pm 1.2$ & $8.8 \pm 1.3$ & 12.7 & $<.01$ & $8.5 \pm 1.2$ & $8.5 \pm 1.2$ & -1.4 & .81 \\
\hline
\end{tabular}

Categoric variables are presented as frequency (\%); continuous variables are presented as mean \pm standard deviation when they were normally distributed or as median (IQR) otherwise. Hemostasis time corresponds to the time lapse between protamine administration after CPB discontinuation and chest closure. ROTEM, Rotational thromboelastography; Std Diff, standardized difference; $S D$, standard deviation; $C P B$, cardiopulmonary bypass; $A A R$, ascending aorta replacement; $A V R$, aortic valve replacement; $C A B G$, coronary artery bypass graft; $R B C$, red blood cell; $F F P$, fresh-frozen plasma; $H b$, hemoglobin.

unit vs $0.0[0.0-2.0]$ unit, $P=.04)$ transfused. There was a decreased requirement in rFVIIa use $(11 \%$ vs $4 \%$, $P=.04)$ and an increased use of DDAVP $(6 \%$ vs $16 \%, P=.02$ ). Blood loss at 12 hours and 24 hours was similar between the 2 groups. ROTEM was significantly associated with less massive transfusion $(21 \%$ vs $11 \%, P=.04)$ and shorter ICU time (44.0 [36.3-49.0] hours vs 32.0 [27.0-39.0] hours, $P<.01)$. There was no difference in regard to perioperative mortality (4\% vs $3 \%, P>.99)$ and reexploration for bleeding $(12 \%$ vs $10 \%, P=.68)$. Moreover, as illustrated in Table 4 , other perioperative complications were comparable between groups.

\section{Sensitivity Analysis}

The semisaturated propensity match yielded 95 pairs of patients from whom standardized differences of 2 demographic and operative variables were greater than $20 \%$ and 22 greater than $10 \%$ (Figure E1). Regarding primary outcome, the rate of RBCs transfusion was similar between both groups (58\% vs $47 \%, P=.15$ ) (Figure 2). Secondary outcomes were mitigated as ROTEM remained associated with less units of RBCs transfused (1.0 [0.0-4.0] unit vs $0.0[0.0-2.0]$ unit, $P=.07)$ and greater DDAVP (5\% vs $18 \%, P<.01)$ use, but showed similar FFP $(0.0[0.0-4.0]$ unit vs $0.0[0.0-2.0]$ unit, $P=.31)$ and rFVIIa $(5 \%$ vs $4 \%, P>.99$ ) requirements (Table E3). Furthermore, PCC 
TABLE 3. Perioperative blood loss and transfusion

\begin{tabular}{|c|c|c|c|}
\hline Variable & Routine $(\mathrm{N}=112)$ & $\operatorname{ROTEM}(N=112)$ & $P$ value \\
\hline Blood loss in $12 \mathrm{~h}(\mathrm{~mL})$ & $1200(895.0-1975)$ & $1160(890.0-1757)$ & .36 \\
\hline Blood loss in $24 \mathrm{~h}(\mathrm{~mL})$ & $1475(1065-2210)$ & $1377(1030-2025)$ & .35 \\
\hline $\mathrm{Hb} 24 \mathrm{~h}$ after index surgery $(\mathrm{g} / \mathrm{dL})$ & $9.8 \pm 1.4$ & $9.5 \pm 1.6$ & .07 \\
\hline $\mathrm{RBC}$ transfusion & $64(57)$ & $51(46)$ & .08 \\
\hline Median number of units (cohort) & $1.0(0.0-4.0)$ & $0.0(0.0-2.0)$ & .03 \\
\hline Median number of units (exposed) & $3.5(2.0-6.0)$ & $2.0(2.0-4.0)$ & .09 \\
\hline FFP transfusion & $43(38)$ & $32(29)$ & .12 \\
\hline Median number of units (cohort) & $0.0(0.0-4.0)$ & $0.0(0.0-2.0)$ & .04 \\
\hline Median number of units (exposed) & $6.0(4.0-8.0)$ & $4.0(4.0-7.5)$ & .05 \\
\hline Platelets transfusion & $61(53)$ & $54(48)$ & .35 \\
\hline Median number of units (cohort) & $5.0(0.0-10.0)$ & $0.0(0.0-10.0)$ & .48 \\
\hline Median number of units (exposed) & $10(5.0-15.0)$ & $10(5.0-15.0)$ & 69 \\
\hline Cryoprecipitate transfusion & $31(28)$ & $29(26)$ & .76 \\
\hline Exposure to $\geq 1$ allogeneic blood product & $75(67)$ & $67(60)$ & .27 \\
\hline Volume of allogeneic blood products (cohort) (mL) & $575.0(0.0-2357)$ & $300.0(0.0-1605)$ & .10 \\
\hline Volume of allogeneic blood products (exposed) $(\mathrm{mL})$ & $1720(550.0-3520)$ & $1100(550.0-2120)$ & .11 \\
\hline Prothrombin complex concentrate & $5(5)$ & $12(11)$ & .07 \\
\hline DDAVP & $7(6)$ & $18(16)$ & .02 \\
\hline RFVIIa & $12(11)$ & $4(4)$ & .04 \\
\hline
\end{tabular}

Categoric variables are presented as frequency (\%); continuous variables are presented as mean \pm SD when they were normally distributed or as median (IQR) otherwise. Median number of units are presented for the whole cohort and for the patients who were exposed to the specific allogeneic product. ROTEM, Rotational thromboelastography; $H b$, hemoglobin; $R B C$, red blood cells; FFP, fresh-frozen plasma; DDAVP, desmopressin; $r F V I I a$, recombinant factor VIIa.

use $(5 \%$ vs $11 \%, P=.07)$ was augmented in the ROTEM group. Finally, the same rate of massive transfusion $(13 \%$ vs $15 \%, P=.67$ ) occurred in both groups, but ICU time

TABLE 4. Postoperative clinical outcomes

\begin{tabular}{|c|c|c|c|}
\hline Variable & $\begin{array}{c}\text { Routine } \\
(\mathrm{N}=112)\end{array}$ & $\begin{array}{l}\text { ROTEM } \\
(\mathbf{N}=112)\end{array}$ & $\begin{array}{c}P \\
\text { value }\end{array}$ \\
\hline Stroke & $6(5)$ & $7(6)$ & .78 \\
\hline Myocardial infarction & $5(5)$ & $4(4)$ & $>.99$ \\
\hline Acute kidney injury & $9(8)$ & $14(13)$ & .27 \\
\hline Reexploration & $12(11)$ & $12(11)$ & $>.99$ \\
\hline Tamponade & $7(6)$ & $4(4)$ & .35 \\
\hline New-onset atrial fibrillation & $25(22)$ & $34(30)$ & .18 \\
\hline Renal failure requiring dialysis & $6(5)$ & $6(5)$ & $>.99$ \\
\hline Postoperative shock & $3(3)$ & $5(5)$ & .72 \\
\hline Sepsis & $2(2)$ & $0(0)$ & .50 \\
\hline $\begin{array}{l}\text { Massive transfusion } \\
\text { (>20 units) }\end{array}$ & $23(21)$ & $12(11)$ & .04 \\
\hline Total ventilation time (h) & $7.75(5.17-14.0)$ & $6.17(4.50-12.0)$ & .06 \\
\hline Total ICU time (h) & $44.0(36.3-49.0)$ & $32.0(27.0-39.0)$ & $<.01$ \\
\hline $\begin{array}{l}\text { Total hospital length } \\
\text { of stay (d) }\end{array}$ & $6.0(5.0-8.0)$ & $6.0(5.0-9.0)$ & .59 \\
\hline Mortality & $7(6)$ & $4(4)$ & .35 \\
\hline
\end{tabular}

Categoric variables are presented as frequency $(\%)$; continuous variables are presented as mean $\pm \mathrm{SD}$ when they were normally distributed or as median (IQR) otherwise. ROTEM, Rotational thromboelastography; ICU, intensive care unit.
(44.0 [28.0-50.0] hours vs 33.0 [27.0-37.0] hours, $P<.01$ ) was shorter with ROTEM (Table E4).

\section{DISCUSSION}

Several studies have shown that the implementation of a thromboelastometry-oriented algorithm for the management of perioperative bleeding in cardiovascular surgeries is associated with a decrease in the use of allogeneic blood products, less unplanned reexploration surgery, lower costs of care, and reduced postoperative morbidity and mortality. ${ }^{15-17,19-23}$ However, previously published randomized control trials had a strictly enforced bleeding management protocol and therefore did not reflect authentic practice. ${ }^{16,17}$ Furthermore, only one focused exclusively on complex aortic procedures. ${ }^{16,17}$

The purpose of this study was to compare perioperative transfusion practice in our center before and after the implementation of a ROTEM-guided protocol for postoperative bleeding management in patients undergoing elective and urgent complex aortic surgery. Ultimately, the goal was to evaluate the effect of POC testing in a real-world setting and assess the immediate impact on surgical practice. Findings from this study demonstrated that the introduction of a ROTEM-based algorithm may lead to a decrease in erythrocytes transfusion requirements for complex aortic operations. 


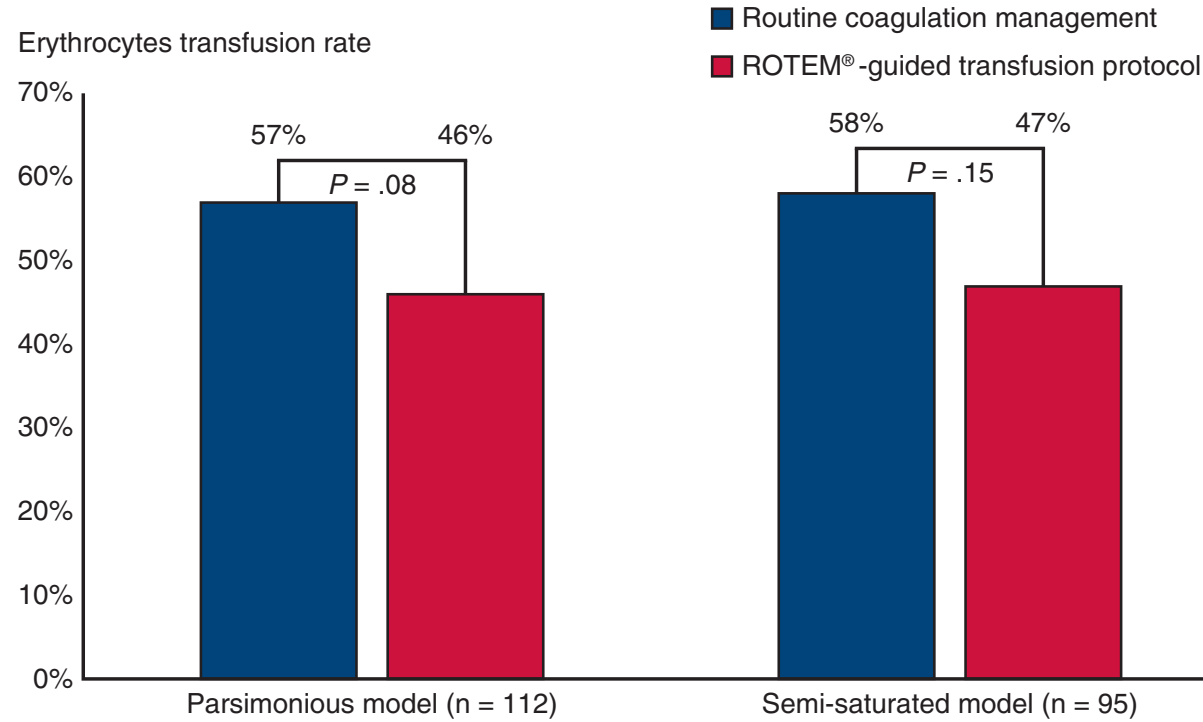

FIGURE 2. The effect of ROTEM-guided transfusion protocol on erythrocytes transfusions. ROTEM, Rotational thromboelastography.

As our results indicate, RBC administration seems lower after ROTEM implementation. After sensitivity analysis, the need for at least $1 \mathrm{RBC}$ transfusion was similar between the 2 groups, but the reduction in the number of units of RBC was still present. Meta-analyses on the subject identified ROTEM as superior to standard coagulation testing in cardiac surgery with regard to quantity of blood products transfused and postoperative outcomes. ${ }^{15,23}$ ROTEM has many theoretic advantages over standard coagulation testing, notably shorter turnaround times (10-25 minutes vs 90-120 minutes) and a thorough understanding of coagulation mechanisms by separating different component of the coagulation cascade (extrinsic pathway, intrinsic pathway, and platelet function). It was also shown to be superior for predicting subsequent bleeding and reexploration. ${ }^{12-14}$ Yet, the data presented here do not reflect those advantages as clearly, and the difference observed could as well be attributed to different patient mix, changes in practice, or other confounding factors. The liberality of implementation of the algorithm, allowing the surgeons to divert from the protocol at any point during coagulation management, is a potential major culprit of the loss of power of our study, especially because data were collected in the first 18 months after implementation.

The possible decrease in transfusion of allogeneic blood products was replaced by an increase in the use of PCC and DDAVP. Although the efficacy and safety of these products is still not clearly demonstrated, they have been found superior to transfusions of allogeneic blood products for stopping the bleeding when specific hemostatic derangements are present, namely, platelet dysfunction for DDAVP and coagulation factor deficiency or dysfunction for PCC. ${ }^{11,24,25}$ Therefore, when combined with POC testing, the use of these agents represents a more targeted approach for coagulation management. There was no fibrinogen concentrate given at our institution because of its limited availability in Canada at the time the study took place.

ICU time was more than $25 \%$ shorter in the ROTEM group, in association with slightly lower ventilation times. Although the clinical implication of such findings is presently unclear, the continuous quality and performance improvement in intensive care could explain this difference between both groups. Reduced transfusions and associated volume overload or faster turnaround times of ROTEM analysis could potentially explain this difference, but no conclusion can be drawn at this point.

An interesting observation is the reduction in the number of patients receiving rFVIIa. This drug should be used only as rescue therapy for intractable bleeding, because it is suspected to be independently associated with a higher rate of thromboembolic complications, and even higher mortality. ${ }^{26-28}$ A decrease in its use not only would be beneficial for the patients' recovery but also presents the potential advantage of lowering economic burden of surgical patients, considering the high cost of rFVIIa. Yet, because this reduction was not correlated in the semisaturated model and is not reproduced in the other studies, it remains hypothetical. ${ }^{15,23}$

Besides the potential for reduced transfusions, our findings revealed equally excellent postoperative clinical outcomes and mortality. This may be explained by multiple factors, such as the small size of the cohort and the low rate of events in both groups. A large proportion of perioperative blood products being administered empirically, a reduction 
in the numbers, and rate of allogeneic blood products transfusion without deterioration in patients' clinical outcomes represent a clear improvement in the practice. ${ }^{4,29}$

\section{Study Limitations}

The retrospective nature of the study and the single-center approach have potential shortcomings that have been well described. The discrepancy between the propensity score models, resulting in smaller cohorts and revealing dissimilarities between both groups, made the benefits directly related to ROTEM difficult to establish and must be acknowledged. Natural progression of surgical and perioperative care and institutional changes in the management or unsteady patient mix, even in the short timeframe of the study (ie, 3 years), all could have partly accounted for the observed difference attributed to the new transfusion protocol. In this study, standardized differences greater than $20 \%$ were considered significant. This should be taken with caution because recent recommendations advocate to seek standardized differences less than $10 \%{ }^{30}$ As noted earlier, the study represents only the data collected in the first 18 months after implementation of the ROTEM-guided protocol. During that time, ROTEM analyses constituted a new tool for most operative teams and the protocol was not strictly enforced into practice. This may have affected the association between the ROTEM-guided protocol implementation and the number of blood components administered. Because we could not correlate, through our database, the transfusions given with the results from ROTEM analysis, we are unable to assess the level of compliance of the surgical team to the new algorithm. Finally, results regarding secondary outcomes should be considered as exploratory because the effect of ROTEM on primary outcome did not reach statistical significance.

\section{CONCLUSIONS}

In a real-world setting, the implementation of a ROTEMbased transfusion protocol for perioperative bleeding management in patients undergoing complex aortic procedures could potentially reduce erythrocytes transfusion, mainly in terms of the absolute number of RBC units needed postoperatively. Our findings highlight the fact that patient selection, strict implementation of the algorithm, and concerted effort of all team members involved in bleeding management are necessary to obtain a significant improvement in the use of blood products after complicated cardiac surgery. Evaluating the impact of the implementation on a more remote time frame may allow to dissipate the initial discordance to protocol and increase the association between ROTEM and bleeding management changes in practice.

\section{Conflict of Interest Statement}

Authors have nothing to disclose with regard to commercial support.

\section{References}

1. de Figueiredo LF, Coselli JS. Individual strategies of hemostasis for thoracic aortic surgery. J Card Surg. 1997;12(2 Suppl):222-8.

2. Woodman RC, Harker LA. Bleeding complications associated with cardiopulmonary bypass. Blood. 1990;76:1680-97.

3. Chu MW, Losenno KL, Moore K, Berta D, Hewitt J, Ralley F. Blood conservation strategies reduce the need for transfusions in ascending and aortic arch surgery. Perfusion. 2013;28:315-21.

4. Kilic A, Whitman GJ. Blood transfusions in cardiac surgery: indications, risks, and conservation strategies. Ann Thorac Surg. 2014;97:726-34.

5. Christensen MC, Dziewior F, Kempel A, von Heymann C. Increased chest tube drainage is independently associated with adverse outcome after cardiac surgery. J Cardiothorac Vasc Anesth. 2012;26:46-51.

6. Karkouti K, Wijeysundera DN, Yau TM, Beattie WS, Abdelnaem E, McCluskey SA, et al. The independent association of massive blood loss with mortality in cardiac surgery. Transfusion. 2004;44:1453-62.

7. Marik PE, Corwin HL. Efficacy of red blood cell transfusion in the critically ill: a systematic review of the literature. Crit Care Med. 2008;36:2667-74.

8. Horvath KA, Acker MA, Chang H, Bagiella E, Smith PK, Iribarne A, et al. Blood transfusion and infection after cardiac surgery. Ann Thorac Surg. 2013;95:2194-201.

9. Vivacqua A, Koch CG, Yousuf AM, Nowicki ER, Houghtaling PL, Blackstone EH, et al. Morbidity of bleeding after cardiac surgery: is it blood transfusion, reoperation for bleeding, or both? Ann Thorac Surg. 2011;91:1780-90.

10. LaPar DJ, Crosby IK, Ailawadi G, Ad N, Choi E, Spiess BD, et al. Blood product conservation is associated with improved outcomes and reduced costs after cardiac surgery. J Thorac Cardiovasc Surg. 2013;145:796-804.

11. Society of Thoracic Surgeons Blood Conservation Guideline Task Force Ferraris VA, Brown JR, Despotis GJ, Hammon JW, Reece TB, et al. 2011 update to the Society of Thoracic Surgeons and the Society of Cardiovascular Anesthesiologists blood conservation clinical practice guidelines. Ann Thorac Surg. 2011;91:944-82.

12. Tanaka KA, Bolliger D, Vadlamudi R, Nimmo A. Rotational thromboelastometry (ROTEM)-based coagulation management in cardiac surgery and major trauma. J Cardiothorac Vasc Anesth. 2012;26:1083-93.

13. Bolliger D, Seeberger MD, Tanaka KA. Principles and practice of thromboelastography in clinical coagulation management and transfusion practice. Transfus Med Rev. 2012;26:1-13.

14. Bolliger D, Tanaka KA. Roles of thrombelastography and thromboelastometry for patient blood management in cardiac surgery. Transfus Med Rev. 2013;27:213-20.

15. Faraoni D, Savan V, Levy JH, Theusinger OM. Goal-directed coagulation management in the perioperative period of cardiac surgery. J Cardiothorac Vasc Anesth. 2013;27:1347-54.

16. Girdauskas E, Kempfert J, Kuntze T, Borger MA, Enders J, Fassl J, et al Thromboelastometrically guided transfusion protocol during aortic surgery with circulatory arrest: a prospective, randomized trial. J Thorac Cardiovasc Surg. 2010;140:1117-11124.e2.

17. Weber CF, Gorlinger K, Meininger D, Herrmann E, Bingold T, Moritz A, et al. Point-of-care testing: a prospective, randomized clinical trial of efficacy in coagulopathic cardiac surgery patients. Anesthesiology. 2012;117:531-47.

18. Casati V, Della Valle P, Benussi S, Franco A, Gerli C, Baili P, et al. Effects of tranexamic acid on postoperative bleeding and related hematochemical variables in coronary surgery: comparison between on-pump and off-pump techniques. J Thorac Cardiovasc Surg. 2004;128:83-91.

19. Karkouti K, McCluskey SA, Callum J, Freedman J, Selby R, Timoumi T, et al. Evaluation of a novel transfusion algorithm employing point-of-care coagulation assays in cardiac surgery: a retrospective cohort study with interrupted time-series analysis. Anesthesiology. 2015;122:560-70.

20. Spalding GJ, Hartrumpf M, Sierig T, Oesberg N, Kirschke CG, Albes JM. Cost reduction of perioperative coagulation management in cardiac surgery: value of "bedside" thrombelastography (ROTEM). Eur J Cardiothorac Surg. 2007;31:1052-7.

21. Pearse BL, Smith I, Faulke D, Wall D, Fraser JF, Ryan EG, et al. Protocol guided bleeding management improves cardiac surgery patient outcomes. Vox Sang. 2015;109:267-79.

22. Karkouti K, Callum J, Wijeysundera DN, Rao V, Crowther M, Grocott HP, et al Point-of-care hemostatic testing in cardiac surgery: a stepped-wedge clustered randomized controlled trial. Circulation. 2016;134:1152-62.

23. Deppe AC, Weber C, Zimmermann J, Kuhn EW, Slottosch I, Liakopoulos OJ, et al. Point-of-care thromboelastography/thromboelastometry-based coagulation management in cardiac surgery: a meta-analysis of 8332 patients. J Surg Res. 2016;203:424-33. 
24. Crescenzi G, Landoni G, Biondi-Zoccai G, Pappalardo F, Nuzzi M, Bignami E, et al. Desmopressin reduces transfusion needs after surgery: a meta-analysis of randomized clinical trials. Anesthesiology. 2008;109:1063-76.

25. Bruce D, Nokes TJ. Prothrombin complex concentrate (Beriplex P/N) in severe bleeding: experience in a large tertiary hospital. Crit Care. 2008;12:R105.

26. Alfirevic A, Duncan A, You J, Lober C, Soltesz E. Recombinant factor VII is associated with worse survival in complex cardiac surgical patients. Ann Thorac Surg. 2014;98:618-24.

27. Gill R, Herbertson M, Vuylsteke A, Olsen PS, von Heymann C, Mythen M, et al. Safety and efficacy of recombinant activated factor VII: a randomized placebo-controlled trial in the setting of bleeding after cardiac surgery. Circulation. 2009;120:21-7.
28. Simpson E, Lin Y, Stanworth S, Birchall J, Doree C, Hyde C. Recombinant factor VIIa for the prevention and treatment of bleeding in patients without haemophilia. Cochrane Database Syst Rev. 2012;CD005011.

29. Snyder-Ramos SA, Mohnle P, Weng YS, Bottiger BW, Kulier A, Levin J, et al. The ongoing variability in blood transfusion practices in cardiac surgery. Transfusion. 2008:48:1284-99.

30. Winger DG, Nason KS. Propensity-score analysis in thoracic surgery: when, why, and an introduction to how. J Thorac Cardiovasc Surg. 2016;151:1484-7.

Key Words: rotational thromboelastometry, aortic surgery, allogeneic, transfusion, coagulation monitoring

Readers who found these articles interesting may also like to read the following papers found in recent and future issues of our sister publications, Seminars in Thoracic and Cardiovascular Surgery and Operative Techniques in Thoracic and Cardiovascular Surgery!

\section{Adult: Perioperative Management}

ORIGINAL MANUSCRIPT: The effect of preoperative anemia on patients undergoing cardiac surgery: a propensity-matched analysis. Fraser Rubens. Semin Thoracic Surg 2018: In press.

ORIGINAL SUBMISSION: Ninety-Day Readmissions of Bundled Valve Patients: Implications for Healthcare Policy. Michael Koeckert. Semin Thoracic Surg 2018: In press.

Editorial Commentary: Bundled Payments for Surgical Care - More Questions Than Answers. Victor Ferraris. Semin Thoracic Surg 2018: In press.

ORIGINAL SUBMISSION: Early Outcomes with Rapid- Deployment Versus Stented Biological Valves: a Propensity-Match Analysis. Anthony Nguyen. Semin Thoracic Surg 2018: 16-23.

Editorial Commentary: Rapid-Deployment Aortic Valve Replacement: Clear Benefits Without a Clear Target Patient Population. Craig M. Jarrett. Semin Thoracic Surg 2018: 24-25.

ORIGINAL SUBMISSION: Randomized Trial of Carnitine for the Prevention of Perioperative Atrial Fibrillation. Farzaneh Dastan. Semin Thoracic Surg 2018: 7-13.

Editorial Commentary: Preventing Postoperative Atrial Fibrillation: is There One Best Option? Vincent R. Conti. Semin Thoracic Surg 2017: $14-15$. 

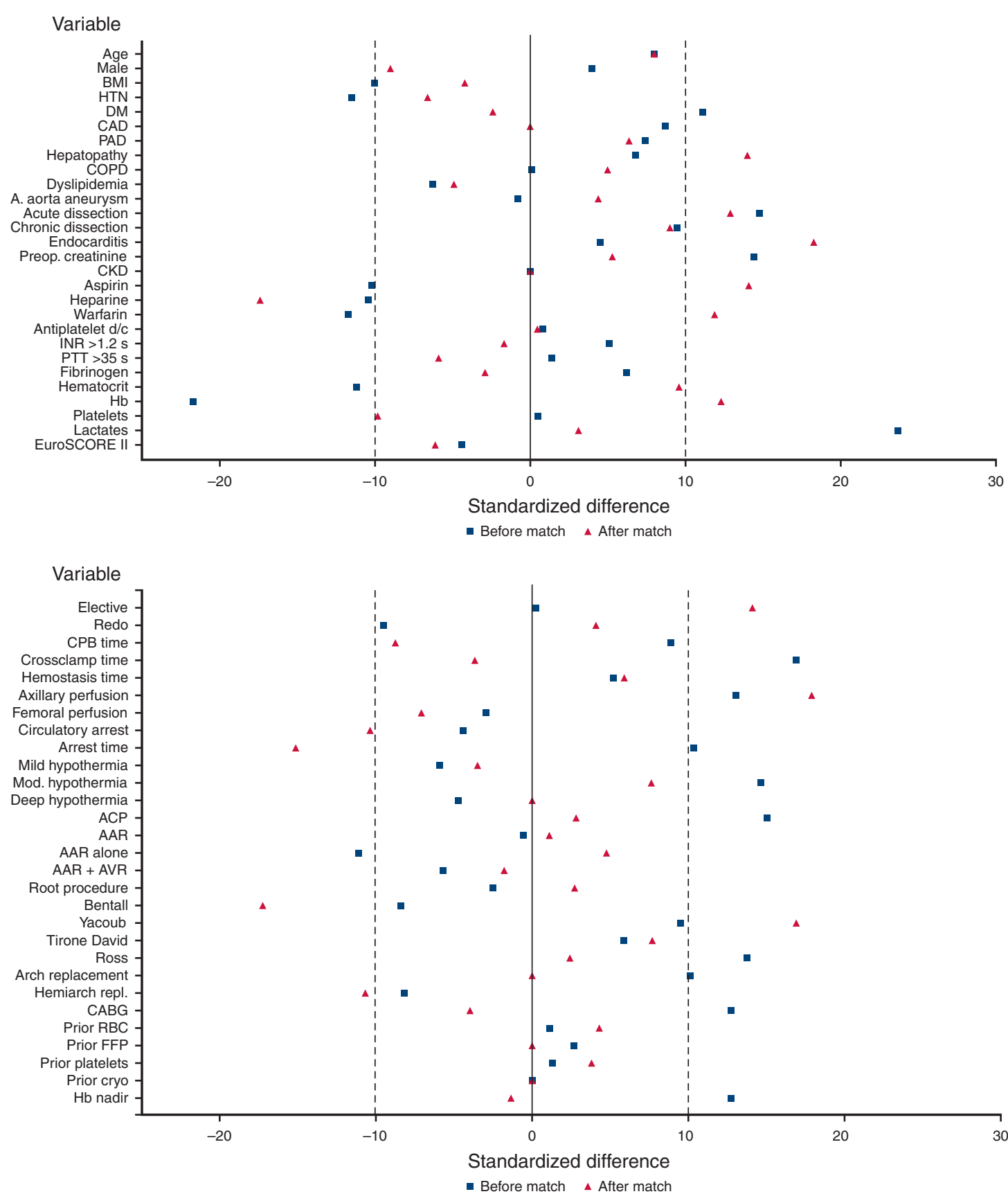

FIGURE E1. Covariance balance plots before and after propensity matching for patients managed according to routine perioperative bleeding management strategy and those receiving POC testing with ROTEM (Tem International GmBH, Munich, Germany). BMI, Body mass index; HTN, hypertension; $D M$, diabetes mellitus; $C A D$, coronary artery disease; $P A D$, peripheral arterial disease; $C O P D$, chronic obstructive pulmonary disease; $C K D$, chronic kidney disease; INR, international normalized ratio; PTT, prothrombin time; euroSCORE, European System for Cardiac Operative Risk Evaluation; $H b$, hemoglobin; $C P B$, cardiopulmonary bypass; $A C P$, anterior cerebral perfusion; $A A R$, ascending aorta remodeling; $A V R$, aortic valve replacement; $C A B G$, coronary artery bypass grafting; $F F P$, fresh-frozen plasma; $R B C$, red blood cell. 
TABLE E1. Baseline characteristics after semisaturated matching

\begin{tabular}{|c|c|c|c|c|}
\hline \multirow[b]{2}{*}{ Variable } & \multicolumn{4}{|c|}{ Propensity score-matched data } \\
\hline & Routine $(\mathrm{N}=95)$ & $\operatorname{ROTEM}(\mathbf{N}=95)$ & Std Diff & $P$ value \\
\hline Age (y) & $57.5 \pm 15.3$ & $58.7 \pm 13.7$ & -8.6 & .56 \\
\hline Male & $62(65)$ & $68(72)$ & 13.6 & .35 \\
\hline BMI $\left(\mathrm{kg} / \mathrm{m}^{2}\right)$ & $27.4 \pm 6.6$ & $28.5 \pm 5.5$ & -18.4 & .21 \\
\hline Arterial hypertension & $52(55)$ & $55(58)$ & 6.4 & .66 \\
\hline Diabetes mellitus & $11(12)$ & $13(14)$ & 6.4 & .66 \\
\hline Coronary artery disease & $23(24)$ & $22(23)$ & 2.5 & .87 \\
\hline Peripheral arterial disease & $9(10)$ & $10(11)$ & 3.5 & .81 \\
\hline Hepatopathy & $2(2)$ & $4(4)$ & 12.1 & .68 \\
\hline COPD & $12(13)$ & $17(18)$ & 14.7 & .31 \\
\hline Dyslipidemia & $42(44)$ & $42(44)$ & 0.0 & $>.99$ \\
\hline Ascending aorta aneurysm & $76(80)$ & $81(85)$ & 13.9 & .34 \\
\hline Acute type A aortic dissection & $10(11)$ & $12(13)$ & 6.6 & .65 \\
\hline Chronic aortic dissection & $3(3)$ & $2(2)$ & 6.6 & $>.99$ \\
\hline Endocarditis & $2(2)$ & $2(2)$ & 0.0 & $>.99$ \\
\hline Preoperative creatinine (mg/dL) & $0.88(0.77-1.05)$ & $0.90(0.75-1.06)$ & -18.4 & .58 \\
\hline Chronic kidney disease & $4(4)$ & $4(4)$ & 0.0 & $>.99$ \\
\hline \multicolumn{5}{|l|}{ Medication } \\
\hline Aspirin & $33(35)$ & $29(31)$ & 10.0 & .54 \\
\hline Heparin & $14(15)$ & $16(17)$ & 5.8 & .69 \\
\hline Warfarin & $11(12)$ & $15(16)$ & 12.3 & .39 \\
\hline Discontinued antiplatelet therapy & $8(8)$ & $7(7)$ & 3.9 & .78 \\
\hline \multicolumn{5}{|l|}{ Coagulation testing } \\
\hline INR $>1.2 \mathrm{~s}$ & $7(7)$ & $12(13)$ & 17.6 & .23 \\
\hline $\mathrm{PTT}>35 \mathrm{~s}$ & $7(7)$ & $6(6)$ & 4.2 & .77 \\
\hline Fibrinogen (mg/dL) & $363 \pm 84.5$ & $418 \pm 124$ & -5.2 & $<.01$ \\
\hline \multicolumn{5}{|l|}{ Hemogram } \\
\hline Hematocrit (\%) & $39.7 \pm 4.1$ & $39.8 \pm 4.3$ & -1.8 & .90 \\
\hline $\mathrm{Hb}(\mathrm{g} / \mathrm{dL})$ & $13.6 \pm 1.6$ & $13.5 \pm 1.5$ & 5.8 & .69 \\
\hline Platelets (cells $/ \mu \mathrm{L}$ ) & $192.7 \pm 54.2$ & $203.8 \pm 71.0$ & -17.5 & .23 \\
\hline Lactates (mmol/L) & $0.9(0.6-1.1)$ & $0.9(0.7-1.1)$ & -30.5 & .09 \\
\hline euroSCORE II (\%) & $6.79 \pm 6.31$ & $7.71 \pm 9.38$ & -11.5 & .43 \\
\hline
\end{tabular}

Categoric variables are presented as frequency (\%); continuous variables are presented as mean \pm SD when they were normally distributed or as median (IQR) otherwise. ROTEM, Rotational thromboelastography; Std Diff, standardized difference; BMI, body mass index; COPD, chronic obstructive pulmonary disease; INR, international normalized ratio; PTT, partial thromboplastin time; $H b$, hemoglobin; euroSCORE, European System for Cardiac Operative Risk Evaluation. 
TABLE E2. Operative characteristics after semisaturated matching

\begin{tabular}{|c|c|c|c|c|}
\hline \multirow[b]{2}{*}{ Variable } & \multicolumn{4}{|c|}{ Propensity score-matched data } \\
\hline & $\overline{\text { Routine }(\mathrm{N}=95)}$ & ROTEM $(\mathbf{N}=95)$ & Std Diff & $\overline{P \text { value }}$ \\
\hline Elective procedure & $77(81)$ & $78(82)$ & 2.7 & .85 \\
\hline Previous cardiac surgery & $17(18)$ & $17(18)$ & 0.0 & $>.99$ \\
\hline CPB time (min) & $113(92.0-165)$ & $123(91.0-166)$ & -0.7 & .68 \\
\hline Aortic crossclamp time (min) & $83.0(63.0-107)$ & $87.0(60.0-120)$ & -0.8 & .56 \\
\hline Hemostasis time (min) & $53.0(44.0-77.0)$ & $57.0(46.0-76.0)$ & -9.4 & .20 \\
\hline Right axillary perfusion & $10(11)$ & $7(7)$ & 11.1 & .45 \\
\hline Femoral perfusion & $7(7)$ & $11(12)$ & 14.4 & .32 \\
\hline Circulatory arrest & $25(26)$ & $28(30)$ & 7.0 & .63 \\
\hline Circulatory arrest time (min) & $4.0 \pm 8.1$ & $4.0 \pm 7.2$ & 0.0 & $>.99$ \\
\hline \multicolumn{5}{|l|}{ Hypothermia } \\
\hline Mild $\left(28.1^{\circ} \mathrm{C}-34^{\circ} \mathrm{C}\right)$ & $70(74)$ & $63(66)$ & -12.9 & .27 \\
\hline Moderate $\left(20.1^{\circ} \mathrm{C}-28^{\circ} \mathrm{C}\right)$ & $20(21)$ & $28(30)$ & -14.5 & .18 \\
\hline Deep $\left(14.1^{\circ} \mathrm{C}-20^{\circ} \mathrm{C}\right)$ & $5(5)$ & $4(4)$ & -1.4 & $>.99$ \\
\hline Antegrade cerebral perfusion & $7(7)$ & $4(4)$ & 13.4 & .35 \\
\hline AAR & $63(66)$ & $53(56)$ & -15.8 & .14 \\
\hline AAR alone & $2(2)$ & $2(2)$ & 0.0 & $>.99$ \\
\hline $\mathrm{AAR}+\mathrm{AVR}$ & $35(37)$ & $31(33)$ & -8.9 & .54 \\
\hline Aortic root procedure & $32(34)$ & $41(43)$ & 12.3 & .18 \\
\hline Bentall & $25(26)$ & $26(27)$ & 2.4 & .87 \\
\hline Yacoub & $5(5)$ & $8(8)$ & 12.5 & .39 \\
\hline Tirone David & $2(2)$ & $7(7)$ & 25.0 & .17 \\
\hline Ross & $9(10)$ & $5(5)$ & -16.2 & .27 \\
\hline Total arch replacement & $3(3)$ & $3(3)$ & 0.0 & $>.99$ \\
\hline Hemiarch replacement & $22(23)$ & $25(26)$ & 7.3 & .61 \\
\hline Concomitant CABG & $16(17)$ & $16(17)$ & 0.0 & $>.99$ \\
\hline \multicolumn{5}{|l|}{ Transfusion before protamine } \\
\hline $\mathrm{RBC}$ & $14(15)$ & $10(11)$ & 7.9 & .38 \\
\hline FFP & $0(0)$ & $2(2)$ & 3.8 & .50 \\
\hline Platelets & $0(0)$ & $1(1)$ & 1.7 & $>.99$ \\
\hline Cryoprecipitate & $0(0)$ & $0(0)$ & 0.0 & $>.99$ \\
\hline $\mathrm{Hb}$ nadir per procedure $(\mathrm{g} / \mathrm{dL})$ & $8.4 \pm 1.2$ & $8.8 \pm 1.3$ & 13.4 & .02 \\
\hline
\end{tabular}

Categoric variables are presented as frequency (\%); continuous variables are presented as mean \pm SD when they were normally distributed or as median (IQR) otherwise. Hemostasis time corresponds to the time lapse between protamine administration after CPB discontinuation and chest closure. ROTEM, Rotational thromboelastography; Std Diff, standardized difference; $C P B$, cardiopulmonary bypass; $A A R$, ascending aorta replacement; $A V R$, aortic valve replacement; $C A B G$, coronary artery bypass graft; $R B C$, red blood cell; $F F P$, fresh-frozen plasma; $H b$, hemoglobin. 
TABLE E3. Perioperative blood loss and transfusion after semisaturated matching

\begin{tabular}{|c|c|c|c|}
\hline Variable & Routine ( $N=95)$ & $\operatorname{ROTEM}(\mathbf{N}=95)$ & $P$ value \\
\hline Blood loss in $12 \mathrm{~h}(\mathrm{~mL})$ & $1180(850.0-1723)$ & $1220(910.0-1760)$ & .56 \\
\hline Blood loss in $24 \mathrm{~h}(\mathrm{~mL})$ & $1375(998.0-1883)$ & $1420(1060-2030)$ & .58 \\
\hline $\mathrm{Hb} 24 \mathrm{~h}$ after index surgery (g/dL) & $9.6 \pm 1.2$ & $9.3 \pm 1.6$ & .18 \\
\hline $\mathrm{RBC}$ transfusion & $55(58)$ & $45(47)$ & .15 \\
\hline Median number of units (cohort) & $1.0(0.0-4.0)$ & $1.0(0.0-2.0)$ & .07 \\
\hline Median number of units (exposed) & $3.0(2.0-5.0)$ & $2.0(1.0-6.0)$ & .23 \\
\hline FFP transfusion & $31(33)$ & $26(27)$ & .43 \\
\hline Median number of units (cohort) & $0.0(0.0-4.0)$ & $0.0(0.0-2.0)$ & .31 \\
\hline Median number of units (exposed) & $4.0(4.0-6.0)$ & $4.0(2.0-4.0)$ & .22 \\
\hline Platelets transfusion & $49(52)$ & $46(48)$ & .66 \\
\hline Median number of units (cohort) & $5.0(0.0-10.0)$ & $0.0(0.0-10.0)$ & .99 \\
\hline Median number of units (exposed) & $5.0(5.0-10.0)$ & $10.0(5.0-15.0)$ & .24 \\
\hline Cryoprecipitate transfusion & $18(19)$ & $25(26)$ & .23 \\
\hline Exposure to $\geq 1$ allogeneic blood product & $66(70)$ & $57(46)$ & .17 \\
\hline Volume of allogeneic blood products (cohort) $(\mathrm{mL})$ & $600.0(0.0-2220)$ & $300.0(0.0-1340)$ & .19 \\
\hline Volume of allogeneic blood products (exposed) $(\mathrm{mL})$ & $1275(550.0-2612)$ & $1110(475.0-2755)$ & .59 \\
\hline Prothrombin complex concentrate & $2(2)$ & $17(18)$ & $<.01$ \\
\hline DDAVP & $5(5)$ & $17(18)$ & $<.01$ \\
\hline RFVIIa & $5(5)$ & $4(4)$ & $>.99$ \\
\hline
\end{tabular}

Categoric variables are presented as frequency (\%); continuous variables are presented as mean \pm SD when they were normally distributed or as median (IQR) otherwise. Median number of units are presented for the whole cohort and for the patients who were exposed to the specific allogeneic product. ROTEM, Rotational thromboelastography; $H b$, hemoglobin; $R B C$, red blood cells; $F F P$, fresh-frozen plasma; DDAVP, Desmopressin; $r F V I I a$, recombinant factor VIIa.

TABLE E4. Postoperative clinical outcomes after semisaturated matching

\begin{tabular}{lccr}
\hline \multicolumn{1}{c}{ Variable } & Routine $(\mathbf{N}=\mathbf{9 5})$ & ROTEM $(\mathbf{N}=\mathbf{9 5})$ & P value \\
\hline Stroke & $4(4)$ & $7(7)$ & .35 \\
Myocardial infarction & $2(2)$ & $3(3)$ & $>.99$ \\
Acute kidney injury & $5(5)$ & $10(11)$ & .28 \\
Reexploration & $8(8)$ & $9(10)$ & .79 \\
Tamponade & $5(5)$ & $4(4)$ & $>.99$ \\
New-onset atrial fibrillation & $20(21)$ & $27(28)$ & .24 \\
Renal failure requiring dialysis & $2(2)$ & $6(6)$ & .28 \\
Postoperative shock & $4(4)$ & $4(4)$ & $>0(0)$ \\
Sepsis & $1(1)$ & $14(15)$ & .99 \\
Massive transfusion (>20 units) & $12(13)$ & $6.3(4.5-10.4)$ & .32 \\
Total ventilation time (h) & $7.8(4.8-14.0)$ & $33.0(27.0-37.0)$ & .67 \\
Total ICU time (h) & $44.0(28.0-50.0)$ & $6.0(5.0-8.0)$ & .31 \\
Total hospital length of stay (d) & $6.0(5.0-8.0)$ & $5(5)$ & .74 \\
Mortality & $3(3)$ & .01 \\
\hline
\end{tabular}

Categoric variables are presented as frequency (\%); continuous variables are presented as mean \pm SD when they were normally distributed or as median (IQR) otherwise. ROTEM, Rotational thromboelastography; ICU, intensive care unit. 